\title{
Representaciones, modelos de acción institucional y transformación sobre el sujeto sin hogar. El caso del "Programa de atención a personas en situación de calle" en Temuco
}

\section{Representations, models of institutional action and transformation on the homeless individual. The case of the "Programme for the care of street-people" in Temuco}

Héctor Muñoz C. ${ }^{1}$

Aceptación: 20 noviembre 2009

Aprobación: 27 abril 2010

\section{RESUMEN}

Este es un intento por reflexionar acerca del tratamiento y abordaje de la pobreza extrema por parte de la institucionalidad local en la ciudad de Temuco, Chile, a partir de un acercamiento antropológico realizado durante el año 2004 al participar dentro del "Programa de atención a personas en situación de calle" de la Municipalidad de Temuco y a las organizaciones e instituciones que conforman la "Red de apoyo a la persona en situación de calle" que se aglutina en torno al programa mencionado. Las reflexiones de este artículo derivan de la labor desarrollada al interior del programa, la observación etnográfica y de entrevistas realizadas a funcionarios y operadores de las instituciones que conforman esta red. El carácter de esta reflexión dice relación con la identificación de representaciones que orientan el accionar institucional, en este caso representaciones en torno a las personas denominadas en "situación de calle". Estas representaciones han permitido ir conociendo de mejor manera el entramado conceptual presente en la red de apoyo, que en este caso determina una forma de actuar y enfrentarse a la realidad y particularmente a la problemática de la marginalidad y la pobreza extrema. En este sentido existe un abordaje, desde la disciplina, de la lógica de los discursos y las prácticas que la sociedad genera en torno a la condición de marginalidad presente en sujetos y grupos, así como el tipo de relaciones e interacciones que se generan a partir de esto.

Palabras clave: Pobreza, marginalidad, representaciones sociales, control social.

\section{ABSTRACT}

This is an attempt to reflect about the extreme poverty treatment and approach from Temuco city local institutions. This approach was carried out in 2004 while participating of the "Homeless people attention program" from the Temuco Municipality and the organizations and institutions that form the "Homeless people support network" which gather around the previously named program. The reflections made in this article draw from the work carried out inside the program, ethnographic observation and interviews made to the network's participant institutions officials and operators. The character of this reflection is related to the identification of representations that guide institutional actions, in this case, representations that have led to a better knowing of the conceptual framework used by the support network, which in this case determines a course of action and a manner of facing the reality and particularly the problem of marginalization and extreme poverty. In this sense, there is an approach, from the discipline, the logic of discourses and practices that the society generates around the marginalized status in individuals and groups as well as the type of relationships and interactions that are generated by this.

Key words: Poverty, marginality, social representations, social control.

Antropólogo, Licenciado en Antropología, Universidad Católica de Temuco. hector_m_c@yahoo.es 


\section{Introducción}

A pesar del importante crecimiento y desarrollo económico de nuestro país, este no tiene un correlato equivalente en el desarrollo social. A pesar de que en las estadísticas oficiales el número de personas pobres ha disminuido en los últimos años, todavía existe un porcentaje significativo de la población viviendo en situación de pobreza y marginalidad. Las condiciones de pobreza van más allá de las carencias materiales y expresan una desigual distribución de los beneficios del crecimiento económico; hay inequidades en relación con los ingresos, el conocimiento, la información, las oportunidades, el poder, etc. De esta manera la erradicación de la pobreza es uno de los problemas sociales no resueltos $y$, por consiguiente ha sido un eje central en las políticas sociales e institucionales en los gobiernos de los últimos 20 años en nuestro país.

Si bien en nuestro país aún no existe una legislación o un cuerpo de políticas sociales que aborde, dentro de la problemática de la pobreza extrema, específicamente el tema de la población "sin hogar" o "en situación de calle", hablamos de una problemática que, al menos en el discurso, se ha transformado en relevante para ciertos grupos sociales que la toman como preocupación principal. En el contexto de la Región de La Araucanía (centro-sur de Chile) existen experiencias claras y concretas que demuestran lo anterior. Nos referimos a diversas instituciones $\mathrm{y} / \mathrm{u}$ organizaciones que desde al año 1998 aproximadamente se encuentran trabajando en torno a las personas que viven en la calle, quizás la población más afectada por los procesos de desigualdad social. A partir de esto se genera también una instancia en instituciones públicas, en este caso en la Municipalidad de Temuco, a través de la Dirección de Desarrollo Comunitario (Dideco), en donde también aparece la necesidad de responder a esta población prácticamente invisibilizada por la gran mayoría de la sociedad.

La Municipalidad de Temuco se define como una corporación autónoma de derecho publico, con personalidad jurídica y patrimonio propio, cuyo objetivo consiste en consolidar los objetivos nacionales de desarrollo eco- nómico, modernización productiva, equidad y justicia social, además de la conservación de lo recursos naturales.

A raíz de lo anterior surge la política de gestión municipal basada en la equidad, participación social, descentralización, eficiencia, integralidad y coordinación pública. Para el logro de los objetivos de la política de gestión municipal, la municipalidad tiene funciones y atribuciones específicas, las cuales se operacionalizan mediante direcciones, programas y proyectos.

La Dideco es la dirección, dentro de la municipalidad, que establece una relación más directa con los habitantes de la comuna. Es la instancia encargada de abordar las problemáticas sociales y demandas de grupos y organizaciones de la comunidad.

Dentro de esta dirección encontramos el "Programa de apoyo personas en situación de calle". Este proyecto tiene sus inicios en el año 1997 en el Departamento social y el Programa de apoyo a la discapacidad, se incorpora trabajando con personas en la situación descrita, lo que se realiza a través de un acompañamiento a personas sin hogar con el propósito de mejorar o cambiar sus condiciones de vida. Lo anteriormente mencionado generó importante conocimiento humano y metodológico, que permitió avanzar en la formulación de un programa específico. De esta manera, a fines del año 2001 se aprueba por parte del Concejo Municipal un proyecto de inversión denominado "Programa de apoyo para la rehabilitación biopsicosocial de personas abandonadas en la calle", cuyo objetivo es "mejorar las condiciones de vida de las personas de la calle de Temuco, con un enfoque integral, que respete la identidad, dignidad y libertad de las personas". El programa, actualmente denominado "Programa de atención a personas en situación de calle", apunta entonces a apoyar la satisfacción de necesidades básicas de alimentación, hospedaje, salud, previsión, entre las más elementales. La metodología de trabajo se concentra en dos grandes ejes: a) acompañamiento-presencia, que consiste en contactar a la persona, conocerla, indagar en su historia de vida, en sus necesidades e iniciar a través de un plan de intervención la búsqueda conjunta de soluciones; y b) traba- 
jo en red, instancia de encuentro que busca enfrentar el trabajo desde una perspectiva interdisciplinaria, tanto desde lo público como desde lo privado. A su vez, las líneas de acción del programa dice relación con la habilitación biopsicosocial, la sensibilización y trabajo comunitario en red, y por último una línea de sistematización de las experiencias del programa.

El eje de trabajo en red permite además aglutinar y visualizar otras institucionalidades, de diversa índole, que presentan diversos acercamientos, directos o indirectos, con la problemática de las "personas en situación de calle". Las instituciones y organizaciones que han sido abordadas en este acercamiento, además del "Programa de atención a personas en situación de calle" son: Hogar ADULAM; YMCA; Centro de Rehabilitación de Adicciones; Servicio de Psiquiatría de Imperial; Hogar de Cristo; Servicio de Registro Civil de Temuco, y Emaús.

Más allá de la diversidad institucional presente en esta red, es posible encontrar ciertas generalidades que dan pie a un análisis del funcionamiento de las instituciones y de los supuestos que orientan el tratamiento y abordaje de las personas sin hogar.

\section{Marginalidad y reacción institucional}

Aceptando que el fenómeno de la marginalidad es una condición establecida dentro de la sociedad, es decir, una realidad interna de esta, entonces "tendría soluciones también internas, dadas por una cambio en las estructuras" (Avaria, 2003). En este sentido el Estado y la sociedad en general generan estrategias que apuntan a integrar y a mejorar ciertas condiciones de vida de sujetos que aparecen como excluidos desde su situación de pobreza y precariedad. Se definen entonces espacios construidos y/o elaborados para atender las problemáticas y necesidades de estos grupos.

El acercamiento a estas problemáticas sociales, tanto desde la estructura estatal como también desde los discursos institucionales y políticos, se ha producido según Avaria desde una lógica centrada y orientada por conceptos de pobreza y marginalidad, a través de los cuales se intenta comprender, explicar y establecer mecanismos y estrategias orientadas a un cambio, respecto a la situación socioeconómica de los sujetos sociales, "especialmente de aquellos que no forman parte de la estructura funcional" (ibíd). Siguiendo el mismo planteamiento, Mendicoa señala que el objeto central de la política social es la pobreza, asociada "naturalmente, al concepto de necesidad que implica estar privado de algo que es imprescindible" (Mendicoa, 1999: 11).

El programa específico al que nos referimos (y las instituciones que conforman la red y lo circundan), cuyo alcance es restringido por las fronteras territoriales y administrativas propias de una municipalidad, permite sin duda conocer el modo en que se manifiestan las instituciones formales de la sociedad en torno a la pobreza. La reacción de estas frente a una problemática de estas características dice relación con mecanismos instrumentales que permitan el abordaje y superación de situaciones que no responden a los ideales de sociedad sobre los cuales el Estado y sus instituciones se basan. Los mecanismos instrumentales, entonces, apuntan a responder y atender las carencias concretas de grupos e individuos catalogados como "pobres" y/o "carenciados".

El "Programa de apoyo a personas en situación de calle" materializa en parte las políticas y preocupaciones de la municipalidad en torno a la pobreza extrema. Estas acciones apuntan, por un lado, a superar situaciones consideradas negativas para los sujetos, particularmente lo que refiere a carencias y dificultad de acceso a la institucionalidad que pueda suplir esas carencias (documentación, salud, alimentación, techo, entre otras) y, por otro, a responder a las exigencias colectivas que pueda hacer la sociedad local. En este sentido, debemos tener en cuenta de que en estos casos se produce una especie de relación dialógica en la cual las políticas y acciones institucionales nacen a partir de la relación con el entorno en el cual se enmarcan.

En la misma línea se elaboran acercamientos académicos y discursivos en torno a distintos procesos generados desde movimientos sociales, los que hacen visible y logran poner en la opinión pública y política una 
determinada situación social que se presenta como inaceptable para diversos sujetos sociales y que por lo tanto amerita la preocupación y participación de la autoridad estatal y gubernamental, así como de institucionalidades locales. En resumen, las instituciones pueden identificar las problemáticas, o recoger las demandas que los propios grupos sociales le hacen en torno a las mismas.

Sin embargo, debemos hacer la salvedad de que en el caso de los sujetos en "situación de calle" no se trata de un colectivo humano que manifieste o se defina por un aglutinamiento en cuanto a sus exigencias como grupo, sino más bien responden a intereses individuales de supervivencia, generando mecanismos y redes que les permitan tal proceso, pero no necesariamente demandando o exigiendo colectivamente. La existencia de un programa de estas características, enfocado en un colectivo humano particularmente invisibilizado por gran parte de la sociedad, rompe con aquella lógica de las políticas sociales e instituciones de servicio, en la cual se supone un movimiento o fuerza social organizada que influye en la modificación y/o creación de nuevas políticas e instituciones².

En este caso particular es más bien un sector de la sociedad el que en cierta medida hace manifiesta la necesidad de tratar desde los organismos del Estado una problemática como la de los "sujetos en situación de calle". En este caso, instituciones que históricamente se han vinculado a estos grupos de personas, hacían presente, de manera más bien implícita, la importancia y necesidad de abordaje de la pobreza extrema, manifestada en las personas que viven en la calle. Instituciones como el Hogar de Cristo o Emaús se presentan como instancias en las cuales se define una preocupación por la problemática de estos sujetos, sin necesariamente generar abordajes sistemáticos y formalizados.

\section{De la inclusión al control social}

Las condiciones de pobreza de los sujetos "sin hogar" o "en situación de calle", que pueden ser entendidas como una diferenciación intrasocietal, como un reflejo de la inequidad y las dificultades de ciertos grupos para acceder a ciertos beneficios materiales y derechos sociales básicos, se presentan además como condiciones que generan exclusión y marginalidad, esto por la incapacidad de responder a patrones de conducta esperados, los cuales estarían influyendo en los procesos de integración social. En este sentido, la condición marginal se vería precisamente reflejada en la oposición o negación de aquellas características que definen al ciudadano "ideal", en este caso un ciudadano participativo política, social y productivamente.

De acuerdo a lo anterior, y en líneas generales, podemos decir que se parte de premisas básicas sobre la estructuración de nuestra sociedad, en este caso una sociedad donde están integrados aquellos de sus miembros que comparten las expectativas y las pautas de valores generalizadas del grupo, y son catalogados como marginados aquellos que, ya sea por su falta de incorporación al sistema productivo, por dificultades de endoculturación, y por asimilar y/o presentar pautas de valores diferentes a los del grupo, no pueden, no quieren o no se les permite participar en un determinado tipo de actividades del grupo, en este caso actividades consideradas integradoras.

Desde una perspectiva funcionalista podemos advertir que la sociedad se organiza y funciona en base a un orden estructural, y que a su vez todo aquello que no cumple ciertas condiciones sociales para estar integrado a tal estructura es consecuentemente marginado. Sin embargo, la misma sociedad se encarga de crear mecanismos que permitan encauzar aquellas acciones y/o actitudes disfuncionales.

La inclusión social, clave dentro de los objetivos de superación de la pobreza, y en particular en el programa descrito, significa englobar al conjunto de la población objetivo en el sistema de las instituciones sociales. Se refiere tanto al acceso a sus beneficios como a la dependencia del modo de vida individual con respecto a los mismos.

En este sentido, entonces, la pobreza extrema aparece como una condición (multi-

Esto se puede ejemplificar en el caso de los movimientos feministas e indígenas, y las influencias que han ejercido a nivel internacional a partir de sus demandas como colectivos. 
dimensional) de ciertos grupos que los hacen aún más vulnerables en cuanto a los procesos de exclusión social. La exclusión social, y su relación con la pobreza, puede ser entendida así, según Avaria (2003), a partir de tres grandes características, "la primera (...) el no acceso a bienes y servicios básicos. La segunda característica estaría más bien determinada por la dificultad en el acceso a los mercados de trabajo y protección social. La tercera y última, relativa al desigual acceso y ejercicio de derechos humanos, políticos, civiles" (Avaria, A, op. cit.).

La satisfacción de necesidades básicas se transforma entonces en el mecanismo central para que los individuos puedan ser reconsiderados como sujetos más allá de sus condiciones de pobreza. De esta manera el programa tiene la posibilidad de otorgar "vales" de alojamiento, realizar contactos para el acceso a un comedor abierto, en ciertos casos, conseguir elementos de trabajo, etc. De la misma manera, se articulan ciertos lazos institucionales que transforman al sujeto en un caso "derivado", es decir, enviado con el respaldo de la municipalidad y el programa hacia otras instituciones que satisfacen ciertas carencias concretas del sujeto, o pueden hacerse cargo de él de acuerdo a sus características y/o condiciones, como es el caso de la rehabilitación alcohólica, alguna atención específica de salud, obtención de documentos, etc.

Los sujetos comienzan paulatinamente a desenvolverse en una red formal de instituciones, en las cuales van encontrando elementos que les permitan acceder a beneficios y ser finalmente "uno más", reconocido como integrado y con capacidad para desenvolverse en la sociedad.

Los supuestos de inclusión social, de equidad, son centrales en el funcionamiento institucional observado. Estos supuestos se operativizan, como ya se ha mencionado, a través del apoyo institucional a la satisfacción de necesidades básicas de los sujetos, como también en el acceso que estos puedan tener a ciertos servicios e instituciones que finalmente les permita entrar en una lógica de desenvolvimiento más formalizado que el que lo caracteriza en su "vida en la calle".
En estos casos la lógica de la inclusión social, y junto a ella la de la equidad, tiene su génesis indisoluble en su contraparte, la exclusión. Ambas categorías entendidas como un proceso, nos permiten entender de qué manera operan estas políticas institucionales y cuáles son sus reales significaciones en las formas de relación que existe entre la sociedad y aquellos individuos que por sus características, condiciones y situaciones determinantes de su identidad, son de una u otra manera excluidos.

Esta exclusión es necesario caracterizarla como exclusión "en sociedad", más que "de la sociedad" (Lo Vuolo, 1995: 15), pues los sujetos, si bien excluidos, reciben los efectos de la sociedad en la que se desenvuelven. Precisamente estos efectos son los que hacen del individuo un sujeto moldeable y a la vez objeto de control. Esto último se refleja en el funcionamiento del programa y en la forma de relación que se establece con los sujetos en contextos institucionales que inevitablemente tienen una carga de poder que determina una relación asimétrica entre los profesionales y los individuos. Si bien se acepta la premisa de que cualquier individuo puede acceder a los beneficios de este programa, existe un proceso de acercamiento a la persona, a través del cual se comienza a tejer una relación en la que el sujeto es orientado hacia ciertas metas y requisitos que lo hagan merecedor de los beneficios. En cierta manera el sujeto debe ser moldeado de acuerdo a los referentes de "normalidad" que orientan los fines del programa y sus objetivos. La conducta de los sujetos es clave para que este se transforme en un verdadero beneficiario. En este sentido, es que aparecen los modelos de personas que, desde un nivel ideacional, pasan a transformarse en verdaderos mecanismos de integración y validación, puesto que se debe responder a ciertas características modélicas que permitan acceder a bienes, servicios y por consecuencia ser un sujeto "aceptado e integrado". En este sentido, estos mecanismos presentes en las relaciones estructurales de gobierno, y en relaciones interpersonales, aparecen "como una estructura muy sofisticada a la cual los individuos pueden ser integrados bajo una condición: que esa individualidad puede ser moldeada de otra forma y sometida a una serie de patrones muy específicos" (Foucault, 1983: 10). 
Las lógicas particulares que caracterizan al individuo de la calle, discrepan profundamente con las normas conductuales que la sociedad en su conjunto establece para los sujetos, esto, entre otras cuestiones, define una tensión entre quienes son integrados y quienes no. A estas normas deben adecuarse los individuos, de manera tal que logren superar sus situaciones de "carencia y exclusión", las que finalmente definen un ethos particular y diferenciado.

Las normas que definirían a un individuo integrado, a la luz de los elementos entregados por el programa, dicen relación con la satisfacción de necesidades básicas, siendo el mecanismo principal, para esto, la capacidad de consumo de los sujetos. Un individuo es integrado cuando tiene la capacidad de consumir y satisfacer sus propias necesidades, es decir, un individuo autónomo dentro de la lógica del mercado. En este sentido, dentro del programa se apunta a que los beneficiarios puedan acceder a algún trabajo remunerado que le permita una constante fuente de ingresos, de manera de subsistir gracias a su fuerza de trabajo. Mientras esto ocurre, el programa es, como su nombre lo dice, un apoyo en el transcurso de ese proceso al cual se aspira desde los lineamientos institucionales.

El programa presentado, así como la municipalidad, son instituciones formales a través de las cuales la sociedad intenta aglutinarse como un todo ordenado, siguiendo lo que son planteamientos clásicos del funcionalismo en las ciencias sociales, haciendo que las acciones institucionales apunten a mantener una determinada forma de existencia común, donde todo individuo esta finalmente determinado por lo que aquellos condicionamientos establecen. El ideal de orden y aglutinación social se concretiza en el funcionamiento de estas instituciones públicas dependientes de un Estado central que se transforma en el referente común de todo ciudadano, y por lo tanto en la estructura determinante en la existencia común de los grupos e individuos.

\section{Representaciones y marcos ideológicos sobre los sujetos "en situación de calle"}

El sociólogo Morell Blanch (2002) plantea que cualquier sistema de organización so- cial que desee mantenerse y perpetuarse en el tiempo debe contar con un universo simbólico que garantice la aceptación de sus miembros a través de un proceso de interpretación y legitimación de sus resultados, incluso de aquellos que resultan disfuncionales, y entre estos, de modo muy especial la pobreza. Es por ello que las distintas conceptualizaciones que históricamente se han dado de este fenómeno social son el reflejo de teorías sociales más generales que pretenden explicar y justificar un determinado modelo de sociedad. "Teorías que no son comprensibles al margen de la estructura económica y social en las que han surgido y se han desarrollado" (Morell Blanch, 2002: 10).

De esta manera las representaciones debemos entenderlas como productos socioculturales, es decir, estructuras significantes que emanan de la sociedad y que nos informan sobre sus características en un determinado momento de la historia. Estos constructos constituyen un proceso de elaboración y construcción social del sentido de la vida cotidiana.

A partir de lo anterior entonces es posibleafirmar que las políticas y programas institucionales contienen en su descripción y definición representaciones de sujetos hacia los cuales conducen su acción o hacia aquellos a los que atenderán. Nos referimos a "nombres" y/o "categorías" con las cuales se intenta aprehender al individuo, a alguien que -al igual que otros- compartiría características determinadas o condiciones que los hacen definibles como conjunto. En este caso las representaciones sociales deben ser entendidas como aquellas definiciones culturales, conceptos y figuras en torno a los sujetos y las situaciones de las que forman parte, que elaboran las instituciones $\mathrm{y} / \mathrm{u}$ organizaciones para diseñar y aplicar acciones y programas.

Debemos consignar entonces que los abordajes oficiales (institucionales) presentes, en este caso, en torno a la pobreza extrema, tejen conceptualizaciones que permiten definir aquello que se toma como objeto de atención. En este caso es posible afirmar que estas representaciones, tanto sobre los individuos como sobre los grupos, definen formas o modelos de acción, y a la vez definen condicionamientos que permiten superar situaciones definidas como negativas. 
En este sentido, aparecen imágenes recurrentes que imponen una identidad al sujeto de la calle, en este caso dando un énfasis importante a los problemas de alcoholismo, así como se grafica en frases como: "La principal causa del problema de la gente de la calle es el alcohol..." (entrevista Hogar Adulam, mayo 2004) o "cada sociedad tiene sus problemas, y en nuestro caso es el alcohol la causa principal de que la gente esté en la calle" (entrevista Ymca, mayo 2004). Estas expresiones llevan consigo la idea implícita de que el individuo que padece tal problemática no es capaz de superarla, por lo cual es necesario transformarlo a él y su entorno para que lo logre. La atención de los sujetos en los contextos institucionales particulares implica una normatividad a la cual el individuo debe adaptarse para participar dentro de la lógica en la que se inserta, "en la casa deben cumplir tareas y la mantienen como si fuera suya, y además deben ingresar sin alcohol" (Hospedería Hogar de Cristo, junio 2004), "con la municipalidad podemos establecer un doble control, tanto al interior del centro como en el entorno de la persona" (entrevista C.R.A., mayo 2004).

El Estado y sus instituciones (también aquellas de carácter privado) se orientan como instancias, por excelencia, abocadas a mantener un orden y una cohesión social, intentando así superar situaciones y condiciones de los grupos sociales, que puedan generar desequilibrios en el orden ideal planteado en los niveles conceptuales de las instituciones.

Las representaciones en torno a los sujetos no solo definen lo que estos son, sino que por sobre todo permiten acercarnos a "lo que deberían ser". Los elementos negativos que caracterizarían a los individuos en su condición de pobreza extrema, ya sea por sus actitudes, sus formas de desenvolverse, su apariencia, permiten caracterizarlos como una representación de lo "no deseado", por lo tanto algo que debe ser modificado. Estas características negativas se presentan en el discurso como ciertas definiciones conceptuales estigmatizantes de los sujetos, principalmente el de "alcohólico" o "loco", los cuales se repiten en la intención de explicar aquellas características que permiten la existencia de procesos de marginación en las personas. Las referencias al alcoholismo en los individuos de la calle, además de sus características estéticas, se hacen desde parámetros negativos, transformando la imagen del "vagabundo" en un sujeto cuasi despreciable, pero a la vez merecedor de una transformación que le otorgue la posibilidad de un mejor "bienestar".

En el caso del denominado "Programa de atención a la persona en situación de calle" y la red institucional que en torno a él se aglutina, esta opera como una instancia que permitiría a la persona comenzar a desarrollar un proceso de paulatina transformación, en este caso para dejar atrás aquellas características no deseables y tornarse en lo que aparece ideacionalmente como "normal", una figura que es posible homologar a lo que los mecanismos oficiales definen formalmente como un ciudadano, un individuo capaz de participar activamente tanto de la vida sociopolítica formal definida por el Estado y su institucionalidad, como la capacidad de entrar en el juego de la competencia y el consumo de la lógica del mercado. Esto se logra en términos operacionales, otorgando al individuo ciertas herramientas que le permitan transformarse en aquel sujeto esperable. La "red" sería la que entregaría esas herramientas básicas, ya sea a través del Registro Civil en cuanto a documentos legales que acrediten al sujeto, el CRA en cuanto a la rehabilitación de ciertos "vicios" rechazados desde la formalidad social, la posibilidad de hospedarse en un hogar para pernoctar durante el tiempo necesario, etc. Este tipo de funcionamiento busca dar forma a un individuo anteriormente deforme, según los requerimientos sociales para su inclusión.

Las conceptualizaciones, o representaciones, no necesariamente son capaces de alcanzar la complejidad presente en el fenómeno representado, en este caso el de la pobreza extrema. Más bien responden a lógicas que homogenizan un grupo con valoraciones y prácticas propias. Las instituciones, de acuerdo a su lógica definen lo que se considera "pobre" y "pobreza”, con una aproximación epistemológica que no necesariamente concuerda con la complejidad del sujeto-objeto de preocupación. Esto lleva a que muchas veces las instituciones homogenicen a los sujetos en situación de calle como "alcohólicos", como "abandonados" por sus familias, como "locos", etc. 
complementando así la idea de pobreza como algo negativo para los individuos, "a nadie le gusta ver gente pobre en su ciudad o su país, y menos si eso va acompañado de vicios como el alcohol..." (Ymca, mayo 2004), "sacar a la gente de la calle es muy difícil, además deben recibir rehabilitación por el alcohol" (Registro Civil, junio 2004).

"Pobre" aparece como una categoría que define una gran masa de individuos y grupos contrarios y/o problemáticos en función de los referentes institucionales, en resumen, son indicadores y situaciones que se deben mejorar. En consecuencia los abordajes que de esta problemática se realizan están relativamente alejados de lo que representa un ethos cultural particular. Por lo tanto las supuestas soluciones que se proponen a una situación definida como problemática para la sociedad, se elaboran a partir de un patrón de referencia ajeno, que corresponde al modelo social presente en los referentes ideológicos, en este caso del Estado, así como de diversas instituciones que se acercan de diferente manera a la pobreza. En este sentido, cobra relevancia el etnocentrismo en torno al cual se define y visualiza al pobre, en cuanto se mide su integración y aceptación de acuerdo a su semejanza o diferenciación con uno mismo, "uno espera que estas personas tengan las mismas oportunidades que tiene uno" (Hogar de Cristo, mayo 2004).

En este momento histórico, donde uno de los principales referentes conceptuales y accionales de la sociedad es el "desarrollo", asociado a la modernización, se establece una lógica unívoca, sin presentar o aceptar alternativas distintas. Los indicadores para determinar carencias, en cuanto condicionantes de la "pobreza", los cuales en gran medida determinan las formas de abordarla y por lo tanto de atender a aquellos individuos en tal situación, en este caso sujetos que se definen por su condición de carecer de redes sociales y familiares, y más específicamente por carecer de un hogar y "vivir en la calle", miden más bien acceso al consumo. Según la terminología oficial, miden "calidad de vida", entendida esta como optimización de la acumulación y su relación con la distribución.

De acuerdo a lo anterior la "lucha contra la pobreza" no se reduce solamente a un problema de carácter técnico en cuanto a su abordaje o a sus objetivos de dar "mejores oportunidades" o "mejorar la calidad de vida", sino que esta lucha contra la pobreza es también la lucha contra los pobres, la eliminación de la pobreza conlleva la eliminación de los pobres, de su ethos cultural, de su particular forma de ver, estar y desenvolverse en el mundo. Es en resumen la negación de la diversidad interna de la sociedad.

Es posible afirmar en este caso que los procesos de marginación e integración que derivan de problemáticas como la pobreza extrema, no solo se producen en un plano accional, sino que se definen en un nivel ideacional. El universo simbólico en el cual se basa y a partir del cual actúan los organismos e instituciones focalizadas en estas problemáticas, se constituye en una trama conceptual en la que deben desenvolverse diversas identidades.

Lo anterior es posible relacionarlo a las formas o modelos de acción institucional, donde se dejan ver ciertos referentes de mecanismos de control que se establecen al interior de las instituciones, que finalmente intentan modelar al individuo según referentes ajenos a su individualidad. Esto implica la comprensión de los contextos institucionales como contextos cerrados, los cuales presentan una normatividad impuesta, donde los sujetos son finalmente reencauzados de acuerdo a las proyecciones propias de los referentes institucionales, más allá de las vivencialidades particulares de los sujetos.

En el caso de las instituciones abordadas en este acercamiento, es posible afirmar que las representaciones que se generan en función de la pobreza, se transforman en condicionantes, no solo de un funcionamiento específico, sino que además se transforman en mecanismos de integración, por lo cual definen una particular forma de respuesta a las problemáticas, a partir de las cuales las situaciones de pobreza se definen externamente como algo que debe ser transformado, apuntando a un cambio sociocultural, no solo de los sujetos, sino que además de los grupos.

El acercamiento a las instituciones ha permitido comprender los supuestos que se reflejan en las acciones en torno a los indivi- 
duos y su condición de pobreza. Las nociones y perspectivas que se presentan en los discursos institucionales sobre los individuos en situación de calle, no dicen relación con lo que podemos denominar una pobreza de características estructurales, es decir, que la persona por condicionamientos externos es y ha sido pobre, sino que más bien se presenta una noción de lo que podemos llamar "empobrecimiento", es decir, una situación procesual por la cual la persona deriva en tal condición de pobreza. Por lo mismo es posible identificar el supuesto de que la persona, así como llega a esta situación, puede lograr una superación de esta, centrando el análisis de la situación en el sujeto, su historia particular e incluso sus propias decisiones, tal como podemos ver en la siguiente frase: "el estar en la calle pasa por una decisión de la persona, algo le ocurre para que este ahí" (Ymca, mayo 2004).

El carácter causal del "ser marginal" aparece centrado en un elemento clave que pasa a ser el individuo. Es este último, específicamente sus particularidades vivenciales, son las que permitirían o detonarían procesos de marginación y exclusión. Desde esta lógica es el propio individuo quien tendría las capacidades de salir de aquella situación de exclusión. Por lo tanto las instancias institucionales formales estarían presentes en el objetivo de entregar insumos que permitieran al individuo, por sí solo, lograr el proceso de reintegración social, "la gente tiene capacidades para salir de su situación, pero hay que ayudarla también" (Hogar de Cristo, mayo 2004).

\section{Reflexiones finales}

Desde estos acercamientos de causaefecto sobre la situación de pobreza y marginalidad de los individuos es posible advertir la falta de cuestionamientos sobre condicionamientos de carácter estructural que den forma a tales situaciones de exclusión.

En nuestro país las posibilidades de profundización democrática, así como la búsqueda de una estructuración equitativa e igualitaria de la sociedad, aparecen como necesidades e intenciones durante los primeros años de la década de los noventa luego de diecisiete años de dictadura, abriéndose las esperanzas de superar problemáticas socia- les como son la pobreza y la exclusión social. La transición hacia la democracia seria aquel proceso a través del cual se iría gradualmente superando las desigualdades así como fortaleciendo la vida democrática de nuestro país.

Sin embargo, y como lo plantea Bengoa, "existe la percepción de que no ha habido transición en lo profundo de la vida social chilena, desde la restauración conservadora ocurrida en la dictadura" (Bengoa, 2000: 28). Esto ha generado un desarrollo particularmente superficial de los mecanismos democráticos en nuestro país, pues estos han operado exclusivamente en niveles formales de carácter político e institucional, perdurando inalterables las estructuras de un modelo de desarrollo que hasta el momento parece incapaz de superar el mantenimiento y reproducción de una sociedad altamente desigual. La democracia, entonces, se presenta problemática e "incoherente", en la medida en que no ha logrado impactar transversalmente al conjunto de las esferas sociales (y las diversas problemáticas que aquellas representan) y básicamente mantiene su funcionamiento limitado a la formalidad institucional.

Establecer ideológicamente la marca de la pobreza como un estado y/o condición de carencia predominantemente material, supone una conceptualización que acarrea como consecuencia para los grupos en tal condición, el quedar sujetos y dependientes de las instancias de caridad y seguridad social institucionalizada, provengan estas desde el mundo privado y la sociedad civil o desde el Estado. Esto además conduce a visualizar la pobreza como una categoría estática, pasiva, homogénea y ciertamente estigmatizada. Esta idea de pobreza, una sociedad altamente desigual y las dificultades de establecer una democracia profunda y transversal, hace que los pobres se transformen en un sector social con un grado de poder y determinación tan insignificante, que finalmente lo hace altamente funcional a la reproducción y mantenimiento de la desigualdad social, política, económica, etc.

Por otra parte, si aceptamos la invisibilidad e imposibilidad de este particular sector social de ejercer activamente un rol en el funcionamiento del Estado y sus políticas, podemos decir que la pobreza se diluye en cuanto 
categoría sociocultural autodefinible identitariamente, pues al ser un sector social "pasivo" es receptor de las categorizaciones que la institucionalidad haga de él, categorizaciones que finalmente orientan el accionar del Estado. Por lo tanto las posibles demandas básicas que emanen desde el mundo de la pobreza, estarán determinadas a las percepciones ideológicas y posiciones del resto de las clases sociales, que si logran expresar un grado más significativo de poder en las estructuras políticas. Esta invisibilidad sociopolítica pone de manifiesto la condición de marginalidad y exclusión social que de una u otra manera perpetúa y agudiza el fenómeno de la pobreza.

En resumen, es la problemática de la desigualdad social la que en gran medida genera y hace de la pobreza un fenómeno marcado por la exclusión social y la marginalización de grupos o sujetos en base de su condición. Si a lo anterior agregamos la conformación ideológica que la pobreza tiene en la actualidad, sobre todo en las esferas institucionales de poder y decisión, podemos afirmar que en el actual orden sociopolítico los pobres, a pesar de su notable expresión estadística y muchas veces mediática, carecen casi por completo de poder, en términos políticos y decisiona- les, que les permita emerger como una fuerza de actores sociales capaces de influir en las políticas que les competen como sujetos y colectivo.

\section{Bibliografía}

AVARIA, A. (2003), "Políticas sociales: exclusión/inclusión en el mundo indígena". En Revista Mad No 8. En: http://rehue.csociales. uchile.cl/publicaciones/mad/08/paper5.htm

BENGOA, J. (2000), La Desigualdad. Testimonios de la sociedad chilena en la última década del siglo XX. Ediciones Sur. Santiago.

MORELL BLANCH, A. (2002), "Reflexiones en torno a la idea de pobreza". En: La Pobreza, revista Anthropos № 194. Barcelona.

FOUCAULT, M. (1983), "El sujeto y el poder". Edición electrónica en www.philosophia.cl, Escuela de Filosofia Uniersidad ARCIS. Traducción de Santiago Carassale y Angélica Vitale.

LO VUOLO, R. (1995), Contra la exclusión, Ciepp, Buenos Aires.

MENDICOA, G. (1999) Exclusión y Marginación social. Nuevas perspectivas para su estudio. Espacio Editorial. Buenos Aires. 